\title{
Die Lehre vom (Alpenstich〉 - in den nosographischen Untersuchungen von Guggenbühl bis Sticker
}

\author{
Eine Reminiszenz zur Seuchentheorie und Seuchengeschichtsschreibung \\ im 19. und beginnenden 20. Jahrhundert*
}

Von Michael Quick

\author{
In civitate nostra Padvae, et Trevisii et Venetiae \\ 1440 de mense Martii antea \\ et post apparvit plevresis mala \\ qvae erat contagiosa ex qua mvlti ac plvrimi moriebantvr.
}

Giovanni Michele Savonarola

Ein zeitgenössischer locus communis besagt, daß eine plausible Theorie so lange Geltung beanspruchen darf, bis sie falsifiziert wird ${ }^{1}$. Dem ist entgegenzuhalten, daß Krankheitsvorstellungen und Therapiekonzepte von erstaunlicher Langlebigkeit und Überzeugungskraft sein können, auch wenn sie nachweislich auf falschen Grundlagen beruhen, falls sie nur einleuchtende Erklärungen für die Genese oder Ausbreitung rätselhafter Leiden bieten ${ }^{2}$. Die historische Krankheitsforschung hat sich darüber hinaus mit diametral gegenläufigen Phänomenen auseinanderzusetzen: zum einen besteht in Mittelalter und früher Neuzeit die latente Tendenz, einzelne Symptome bzw. unterschiedliche Zeichen einer einzigen Krankheit zu jeweils eigenständigen Leiden zu erheben ${ }^{3}$, auf der anderen Seite begegnet man neueren Versuchen, Krankheitsklassen und -familien zu bilden, d.h. nosologische Strukturen zu schaffen, die vereinheitlichen, was von durchaus unterschiedlicher Ätiologie bestimmt ist ${ }^{4}$. Wenn im folgenden ein fehlgeschlagener Versuch der Seuchendarstellung und -systematisierung vorgestellt und die Entwicklung einer medizinischen Theorie in ihren Grundlagen und ihrer Verbreitung nachgezeichnet wird, so ist dies nicht als ein unkonstruktiver Beitrag zu einer weitgehend überflüssigen Geschichte ärztlicher Irrtümer zu verstehen: an Beispielen für wissenschaftliche Sackgassen und irreführende Abwege

\footnotetext{
* Das zugrundeliegende Referat «Georg Sticker und die historische Beschreibung des Alpenstichs> wurde am 10.Oktober 1986 der Jahresversammlung der Schweizerischen Gesellschaft für Geschichte der Medizin und der Naturwissenschaften in Bern vorgetragen.
} 
findet sich bereits genug ${ }^{5}$. Vor diesem Hintergrund und im Bewußtsein, da $\beta$ auch die Medizin der Gegenwart ihrer Unzulänglichkeit und Zeitgebundenheit stets eingedenk sein muß, soll vielmehr exemplarisch die frühe Problematik gezeigt werden, wie man Historie für die Epidemiologie nutzbar machen oder aber sie dafür mißbrauchen kann; darüber hinaus soll erneut vor der kritiklosen Übernahme von imponierenden Forschungsergebnissen gewarnt werden, die dazu führen kann, daß Fehler sich trotz ihrer Widerlegung über Dezennien hinweg hartnäckig fortpflanzen können ${ }^{6}$.

Die Tradition als Leitlinie für zukunftsweisende Zielsetzungen: gemäß diesem Motto verstehen auch heute noch bedeutende Epidemiologen Geschichte als unverzichtbaren Bestandteil ihres Faches? ${ }^{7}$. Doch schon zu Beginn des Jahrhunderts war der Internist und Loimologe Georg Sticker ${ }^{8}$ ein unermüdlicher Mahner für stärkere Berücksichtigung der geschichtlichen Erfahrungen in der Seuchenlehre. Kaum eine Infektionskrankheit, deren historischen Verlauf Sticker in seinen akribischen Forschungen nicht berücksichtigt hätte ${ }^{9}$. Beredten Nachweis dafür bietet neben dem gedruckten Werk auch das Arbeitsmaterial. Es ist ein außerordentlicher Glücksfall, daß nach dem Tode des Gelehrten im Jahre 1960 - das war zu Beginn seines 101. Lebensjahres - sein gesamter wissenschaftlicher Nachlaß auf dringendes Anraten von Robert Herrlinger in die Bestände der Universitätsbibliothek Würzburg übernommen werden konnte. Die nach Arbeitsgebieten geordnete Sammlung enthält unter anderem einen Karton mit dem Titelvermerk (Alpenstich〉. Ausgehend von der Stickerschen Dokumentation entstanden die folgenden Grundzüge der historischen Beschreibung des «Alpenstichs - einer nosologischen Chimäre.

In einem 'Wörterbuch des menschlichen Wissens', besser bekannt als «Meyers Konversations-Lexikon`, wird nachstehende Definition der Krankheit gegeben: "Alpenstich, in den Hochgebirgen der Schweiz endemische und in den meisten Frühjahren auftretende, leicht typhös und tödtlich werdende Brustfellentzündung, Folge des südlichen Alpenwindes (Föhns), welche sich 1771 und 1832-33 selbst über einige Theile des nördlichen Deutschlands ausdehnte. Bisweilen erscheinen nach den Anfällen blaue Flecken auf der Haut.»10 Diese Kenntnisse galten im Jahr 1857 somit als Gemeingut, nachdem bereits zwei Jahrzehnte vorher erstmals Aufmerksamkeit auf die Seuche gelenkt worden war.

1838 erscheint bei S. Höhr in Zürich ein Büchlein von gut 120 Seiten mit dem Titel: \Der Alpenstich endemisch im Hochgebirg der Schweiz und seine Verbreitungen>. Sein Autor heißt Johann Jakob Guggenbühl ${ }^{11}$, der damals 22 
Jahre alt ist, Doktor der Medizin und praktizierender Arzt. Als namhafter Verfasser des Vorwortes zeichnet verantwortlich der Arzt, Professor der Philosophie und Berner Ehrenbürger Ignaz Paul Vital Troxler (1780-1866). Er lobt die historischen Studien seines jungen Kollegen, weil sie auf breitem Quellenfundament aufbauten, und empfiehlt die Schrift der Medizin studierenden Jugend, denn: «Die meisten Handbücher, die man in ihre Hände legt, die meisten Vorträge, die man vor ihnen entfaltet, beziehen sich auf eine ins Einzelne und Gewöhnliche versunkene Privatpraxis wie sie die moderne Speculation und Empirie unserer deutschen und welschen Nachbarn diktirt. Die Verkehrtheit und das Verderben dieses Verfahrens fällt in die Augen und hat sich auch hie und da schon praktisch und faktisch fühlbar gemacht.» ${ }^{12}$ War der Autor aber vielleicht schon zu weitsichtig?

Guggenbühls Seuchenchronik umfaßt den Zeitraum von 1550 bis 1837. In diesen knapp $300 \mathrm{Jahren}$ findet er 21 bedeutende Seuchenzüge, von denen er den folgenden besondere Bedeutung beimißt:

1564/65 in Bormio, sich ausbreitend nach Chur, Zürich ${ }^{13}$ und Basel bis hin nach Österreich (Wien), Deutschland (Heilbronn, Augsburg, Kleve a. Rh.) und den Niederlanden;

Beschreibungen der Epidemie(n) durch Conrad Gessner (15161565), Taddeo Duno (1523-1613), Johannes Crato von Krafftheim (1519-1585), Johann Weyer (1515-1588), Rembert Dodoens (1517-1585), u.a.

1652 im Kanton Glarus;

Nachricht darüber gibt Johann Heinrich Tschudi (1670-1729).

1757 ausgehend von Grindelwald (weitere Epidemien u.a. 1750 und 1765), mit Verbreitung nach Nieder-/Oberbipp [BE] bis weiter nach Deutschland und Flandern;

wichtigste Quellen für die Schweiz sind das Totenbuch von Grindelwald, der Bericht eines Amtsarztes namens Kopp, für Flandern die Beschreibung durch Charles-Augustin van der Monde (1727-1762).

1762 in Ormont sowie Aelen und Saanen (alle im Kanton Bern); Berichterstatter ist vor allem Victor Albrecht von Haller (17081777).

1764 in Italien, namentlich in Napoli; Informationen bei Michele Sarcone (1732-1797).

$1765 / 66$ in Lausanne; 
eingehende Angaben macht Samuel Auguste André David Tissot (1728-1797).

1771 in den Kantonen Glarus, Luzern, Nidwalden, Schwyz, Zürich, St.Gallen u.a., des weiteren in Baiern, Württemberg, Sachsen, Preußen, in Hannover, Prag und Wien;

unter den zahlreichen Mitteilungen auch eine von Johann Georg Zimmermann (1728-1795).

1806/07 in den Kantonen Uri, Nidwalden, Glarus und Luzern; Ausdehnung der Seuche über die Schweiz und bis nach Deutschland;

Schriften darüber stammen von Ignaz Paul Vital Troxler und Franz Richli ${ }^{14}$.

1832/33 im Hochtal von Ursern mit Andermatt [UR], im Tal der Reuss, an der Südseite des Vierwaldstättersees bis nach Stans;

beschrieben u. a. von Johann Lucas Schoenlein (1793-1864).

1837 in den Kantonen Graubünden, Bern und Solothurn;

von Guggenbühl selber untersucht.

Der sogenannte (Alpenstich〉 zeigte sich also keineswegs nur im Hochgebirge, sondern auch im Flachland - jedenfalls in Guggenbühls Interpretation -, wenn auch in veränderter Gestalt mit - als «Versätze» bezeichneten Affektionen innerer Organe wie Milz, Leber, Gehirn etc. Sein Einflußgebiet reichte angeblich vom Mittelmeer bis zur Nordsee, vom Jura bis nach Böhmen. Guggenbühls Nachricht über den (Alpenstich〉 wirkt entsprechend sehr eindrucksvoll. Er zeichnet das Bild einer mächtigen, ständig wieder aufflackernden Seuche, «die so oft die stillen Alpenthäler verödete, und zum Schrecken vieler Nationen weit entfernte Gegenden durchlief». ${ }^{15}$

Im übrigen ist es auch Guggenbühl, der die Bezeichnung (Alpenstich〉 überhaupt prägt ${ }^{16}$.

So bestimmend wollten sich seine Vorgänger nicht äußern: «definire non erat facile, propter signorum varietatem magnam» schrieb Taddeo Duno über das bis anhin unbekannte Erscheinungsbild der Epidemie. Und er begründete wie folgt: "Alii enim secundum membranam, alii vero secundum musculos laborare magis videbantur. Nam qui difficiliorem spirationem habebant, dolorem pungentem \& acutum, febrem vehementiorem, pulsum duriorem, cruentum sputum, \& in latus affectum facilius decumbebant, iis membrana proculdubio erat inflammata». Duno schlägt den Terminus der «pleuritis pestilens» vor, ohne jedoch darauf zu bestehen: «dicemus de nominibus non esse contendendum, modo de rebus constet.» ${ }^{17}$ 
In der Folgezeit gebrauchte man gleichwohl andere Namen und Umschreibungen, sowohl die Ärzte als auch die Betroffenen. Der Volksmund nannte die epidemische Krankheit den «heimlichen Stich» oder den «faulichten Stich». "Noch in unserem Jahrhundert wussten alte Leute aus dem Munde ihrer Grossmütter von der Todesfurcht zu erzählen, welche sich der Bewohner des Landes bemächtigte, als es hiess, 〈der böse Stich〉 gehe aus», berichtet August Feierabend anno 1866. Man ängstigte sich wohl auch wegen der bedrohlichen Ungewißheit im Zusammenhang mit der sogenannten «neuen Krankheit» (1817) oder litt gar am «Schwinder», d.h. an Vertigo. Conrad Gessner konstatiert eine "pleuritis maligna, pestilens et contagiosa» (1564), und "pestis pleuritica» lautet ein zweiter Vorschlag, den Taddeo Duno zur gleichen Zeit macht. 〈Pleuropneumonie〉, 〈Faulfieber〉, 〈galliger Seitenstich), «epidemisches faules Gallenfieber〉, (Brustkrankheit), ‘Fäulungsfieber), (hitziges Brustfieber) und viele mehr sollen gebräuchliche Begriffe für die gefürchtete enigmatische Krankheit gewesen sein. Was für Tissot 1766 der «bösartige Seitenstich» ist, heißt bei Troxler 1807 «febris cachectico-typhosa» (bzw. "faulig-typhöses Fieber»), nennt Richli zehn Jahre später eine «Pleuropneumonia biliosa-nervosa, seu putrida» (bzw. «faulige gallige Lungenentzündung») während Brun 1840 von einer «pneumonia erisypelatosa» («rothlaufartige Lungenentzündung») spricht ${ }^{18}$. Im 19. Jahrhundert dominiert anscheinend «die Annahme, daß der Alpenstich ein innerer Rothlaufprozeß, und zwar meist ein Lungenrothlauf sei» ${ }^{19}$. Bekanntlich wechseln Bezeichnungen häufig, doch mußte eine solche Namensdivergenz schon hellhörig machen.

Analog zu dieser Benennungsvielfalt finden sich auch die unterschiedlichsten Krankheitsbilder, die dennoch alle Ausprägungen der nämlichen Seuche sein sollen ${ }^{20}$.

Guggenbühl nennt als gemeinsame Merkmale:

Das Leiden beginne ohne Vorboten mit Kälteschauern und nachfolgendem Hitzegefühl sowie Fieber. Darauf setze das charakteristische, namengebende Stechen im Brustraum ein, oft einseitig lokalisiert, z. T. auch diffus im Bereich der falschen Rippen und bis zum Schlüsselbein und zum Rücken ausstrahlend. Atembeschwerden zeigten sich anfangs kaum, Husten und Sputum seien mäßig. Das Gesicht des Kranken sei gerötet, die Zunge leicht belegt. Der Puls verlaufe weitgehend normal. Nach zwei Tagen wende sich die Lage zum Schlechten: Ekelgefühle und Beschwerden im Intestinalbereich verstärken sich; es komme zu Diarrhoen und Vomitus. Rasch schreite die Entkräftung voran bis zu apathischen Zuständen. Die Respiration werde 
mühsam und rasselnd. Heftige Schweißausbrüche stellten sich ein, die Ĥaut verfärbe sich gelblich. Ödeme oder Petechien könnten sich bilden, der Puls werde schnell und schwach. Zwischen dem fünften und siebten Tag trete nach auffälliger Linderung des Leidens unversehens der völlige Zusammenbruch mit Todesfolge durch Ersticken oder Gehirnschlag ein.

Neben dieser sogenannten «alpinen Form» existierten in der Einteilung nach Tissot (1765) noch eine entzündliche Form mit stark «typhösen» Symptomen und als schlimmste Art die «torpide», das «reine Faulfieber der Alten». ${ }^{21}$

Zur Mortalität bemerkt Guggenbühl weiterhin: «Nach der Sterblichkeit zu urtheilen, gehört die Krankheit zu den schlimmsten Seuchen, die man kennt.» Er wird denn auch nicht müde, das Ausmaß der Epidemien in seinem ganzen Schrecken lebhaft auszumalen. Für ihn gilt auch als sicher, daß es sich um ein übertragbares ¿Übel〉 handelt, mit der Einschränkung, «dass dieser Krankheit kein originäres permanentes Contagium zukommt». [!]

Die Ursachen der Erkrankung glaubt er in unüblichen Witterungswechseln zu finden; ein weiterer Faktor sei die armselige und in der Hygiene äußerst mangelhafte Lebensweise der Alpenbewohner.

Um der Seuche zu entrinnen, gibt er bevorzugt diätetische Ratschläge; daneben empfiehlt Guggenbühl als probate Therapeutica: Brechmittel, möglichst frühzeitig verabreicht, die «Application äußerer Hautreize», z. B. mittels Blasenpflaster, gelinde stuhlfördernde Substanzen, vorzüglich Tamarindenextrakte, sowie Sedativa ${ }^{22}$. In späten Krankheitsphasen mißt er Reizmitteln hohen Wert bei: «Der verschiedentlich nach den Umständen vermischte Kampher unterstützte die Kräfte, verhinderte die Verderbnis der Säfte, unterhielt die Ausdünstung, beförderte den Auswurf und verdient in dieser Krankheit das größte Lob.» - So sein von Tissot übernommenes Urteil. Guggenbühls Mentor Troxler hingegen soll mit Vorliebe Analeptika und «metallische Arzneimittel» wie z.B. Quecksilber verordnet haben. «Diese beiden Arten von Heilmitteln verabreichte Dr. Troxler im Wechsel, und will von einer beträchtlichen Anzahl Kranker auch nicht einen verloren haben.» [Und das bei einer spezifischen Mortalität von bis zu 4\% und einer Letalität bis zu $90 \%$, wie sich aus den Angaben von Guggenbühl und Feierabend errechnen läßt! ${ }^{23}$

Seltene Einmütigkeit herrschte in den Ansichten bezüglich der Phlebotomie bei «Alpenstich >-Patienten. Zu einer Zeit, in der allen Reformversuchen Brissots zum Trotz gerade Pleuritis und Pneumonie Indikationen für Aderla 3 waren ${ }^{24}$, mahnte Gessner in seiner «Descriptio pleuritidis pestilen- 
tis, malignae et contagiosae Martio et Aprili 1564 in urbe Tigurina publice grassantes zur Zurückhaltung beim Purgieren. Auch Haller beispielsweise übte Vorsicht mit seinen kurativen Maßnahmen und mahnte zu einer genauen Unterscheidung der Krankheitsformen. In seiner Beschreibung einer 〈Faulfieberepidemie〉 von 1762 schreibt er ferner: «Die Aderlássen, die warme Diä, der Wein den man den Kranken gab, der Theriak, niedrige und unglaublich heisse Schlafkammern, können das ihrige dazu beygetragen haben, diese Uebel so morderisch zu machen.» ${ }^{25}$ Statt dessen riet Haller zu sauren Getränken, zu Wasser, Kühle, viel reiner Frischluft, Obst, einem leichten Lager usf.

Ein besonderes Anliegen war den Medizinern seit den Erfahrungen Gessners, daß in jedem Krankheitsfalle unverzüglich ein Arzt zu Rate gezogen würde. «Dieses ist bei der ursprünglich täuschenden Gelindigkeit, bei der Unaufmerksamkeit des Volkes in den Gebirgen auf sich selbst, dem oft noch herrschenden Fatalismus - das einzig wahre Mittel nebst der rationellen Behandlung, um bei grössern Epidemien der Verheerung zu steuern, was wir somit der verdienten Beachtung empfehlen.» ${ }^{26} \mathrm{Wie}$ eng jedoch der Handlungsspielraum der Ärzte war, verdeutliche die vehemente Äußerung Troxlers: «Er [Troxler] erklärte dabei die damals sehr üblichen Blasenpflaster meistens für entbehrlich, die Schröpfköpfe für verwerflich, die Brechmittel für schädlich und die Aderlässe als mörderisch.» [! $]^{27}$

Guggenbühls Bemerkungen über den (Alpenstich〉 führen zurück auf Maximilian Stoll (1742-1787), nach dem das Krankheitsbild des «Erysipelas pulmonum) als (Pleuritis biliosa) (daneben auch «Pneumonia biliosa) bzw. (Pleuro-Pneumonia biliosa) neue Beachtung fand. Hinzu tritt der Einfluß Schoenleins. Man vergleiche hierzu die (von Carl Ludwig Reinhard anonym herausgegebenen) Vorlesungsnachschriften über sallgemeine und specielle Therapies, die Schoenlein untergeschoben wurden. Für die Wirkungsgeschichte Guggenbühls erscheint bedeutsam, daß noch nicht in der vierten Auflage oben genannter Skripten (St. Gallen, 1839), wohl aber in der fünften (St.Gallen, 1841) «eine besondere Art von Pneumonie» erstmals aufgeführt wird: «Pneumonia alpina. Der bösartige Stich. Alpenstich.» ${ }^{28}$ Erscheinungsformen, Verlauf, vermeintliche Ätiologie und Vorkommen, Ausgänge und Behandlung werden dabei in Kürze dargelegt, jedoch nicht so dramatisch wie bei Guggenbühl.

Wohl der erste, der die genannten Ergebnisse und Untersuchungen von Guggenbühl nahezu identisch übernimmt, ist Heinrich Haeser (1811-1884), und zwar schon in den Jahren von 1839 bis 1841 . Bei seiner Übersicht zu den 
Volkskrankheiten ${ }^{29}$ beschreibt er immer wieder als Einschub ein «äusserst ausgeprägtes Lungenleiden», das er in Anlehnung an den sogenannten Petechialtyphus als «typhöse Pneumonie» charakterisiert; der Begriff 〈Alpenstich〉 wird synonym dazu gebraucht. Als chronologische Quellendarstellung ${ }^{30}$ ist Haesers Studie auch heute noch interessant, seine überholte Terminologie und Nosologie ist im mikrobiologischen Zeitalter dagegen ohne Bedeutung. Die Überzeugung, mit der er die neue Theorie vom «Stich〉 aufnahm, äußert sich besonders deutlich in folgenden Passagen: «Wir freuen uns, an dieser Stelle noch der vortrefflichen Arbeit gedenken zu können, welche ganz vor kurzem Guggenbühl über diese typhösen Pneumonieen geliefert hat.»- «[...] verbreiteten sich im Frühlinge, besonders in März und April, über ganz Italien und die benachbarten Länder Krankheiten, die wir nach den sehr werthvollen Nachrichten genannter Ärzte [Codronchi, Laelius a Fonte, Colle, Chiocchi] nur für Epidemieen des (Alpenstich) halten können, obschon Guggenbühl derselben für die Schweiz in diesen Jahren nicht erwähnt.» ${ }^{31}$

Ein zweites Mal beschäftigt sich Haeser in seinem medizinhistorischen Lehrbuch von 1845 intensiv mit dem (Alpenstich〉 und hält - ausgehend von der «allgemeine[n] Krankheitsconstitution des 16ten Jahrhunderts» - nicht zuletzt «die typhösen Pneumonien des 16ten Jahrhunderts» sogar einer noch genaueren Beschreibung für wert. Er weist u. a. darauf hin, daß die Ärzte jener Zeit sehr wohl zu Differentialdiagnosen befähigt gewesen wären, daß auch vorhergehende und gleichzeitig verbreitete Krankheiten in Betracht gezogen worden wären. Insbesondere Italien liefere wichtige Anhaltspunkte für die Verbreitung und Erkennung der malignen «Pleuritides erysipelatodes». ${ }^{32}$ Der (Alpenstich〉 wird dem Leser als bedeutsamer Bestandteil der damaligen Epidemien vorgeführt.

Zehn Jahre nach der Darstellung Guggenbühls erhebt sich herbe Kritik an seiner Monographie durch eine Veröffentlichung von Conrad MeyerAhrens, mit dem Titel «Der Stich in den Jahren 1564 und 1565 im Zusammenhange mit den übrigen Epidemieen der Jahre 1562-1566), erschienen in der «Schweizerischen Zeitschrift für Medicin, Chirurgie und Geburtshülfe», daneben auch in Buchform bei F. Schulthess in Zürich, 1848. Dieses Werk ist in Wahrheit eine Studie über die Pest, wohingegen die mysteriöse Pleuritis oder «Brustseuche» nur etwa einen Fünftel des Berichtes ausfüllt.

Meyer-Ahrens legt schonungslos die zahlreichen Oberflächlichkeiten, Trugschlüsse und Fehler dar, die Guggenbühl großzügig in Kauf genommen hat, um seine neue Seuche beweisen zu können. Da sind beispielsweise ganze 
Zitate in den Quellen nicht nachzuweisen, da bringt Guggenbühl - z.T. erhebliche - falsche Datierungen, verwechselt er Autoren, transkribiert unvollständig und teilweise fehlerhaft, übersieht mehrfache Bedeutungen lateinischer Krankheitstermini, unternimmt waghalsige Schlußfolgerungen aus zusammenkonstruierten sogenannten Fakten und dergleichen mehr ${ }^{33}$. Meyer-Ahrens arbeitet wesentlich genauer - er kennt z. B. auch die aufschlußreiche Sammlung der Briefe Conrad Gessners bis ins Detail, beweist überhaupt eine exakte und eindrucksvolle Quellenkenntnis - und er urteilt vorsichtiger. Der Begriff 〈Alpenstich〉 existiert für ihn nicht; er meidet auch die Bezeichnung «Stichepidemie» soweit möglich. Außerdem bemüht er sich um eine grobmaschige Differenzierung in Pleuritis, Pesttyphus, bösartige Katarrhe, Petechialtyphus etc. Darüber hinaus bringt er ganz eindeutig das Abklingen der Pest mit der Zunahme der Pleuritis in Verbindung und sieht umgekehrt die «typhöse Brustseuche» von der Pest abgelöst werden ${ }^{34}$. Diese Erkenntnis war ein Vorläufer der Ergebnisse Stickers.

Zur Kontroverse um die Guggenbühlsche Arbeit ist nachzutragen, daß Meyer-Ahrens sich bereits vorher eine Auseinandersetzung mit seinem Kollegen geliefert hatte. Die 1843 erschienenen «Geschichtlichen Notizen über die Verbreitung des Englischen Schweißes in der Schweiz im Jahre 1529> sind nämlich als Emendation der Berner Inaugurationsschrift Guggenbühls «Der englische Schweiß im Jahre 1529 in der Schweiz〉 (1838) zu verstehen ${ }^{35}$.

Eine Würdigung der Untersuchungen von Meyer-Ahrens wiederum bringt August Wilhelm Theodor Henschel (1790-1856) im dritten Band des von ihm herausgegebenen 〈Janus〉. Diese Rezension birgt einen wichtigen, kritisch weiterführenden Gedanken. Henschel schreibt: «Verf. [...] zeigt [den] genetischen Zusammenhang insbesondere mit der katarrhalischen und typhosen Constitution, die sich zu dieser Zeit in den mannigfaltigsten epidemischen Affectionen der Haut, der Pharyngeal- und Bronchialschleimhaut, in Influenza, Masern, Angina, Pleuritis u.s.w. aussprach: unter diesen tritt nun der Alpenstich, als der Hauptgegenstand dieser Schrift, in Gestalt einer typhösen Pneumonie (wie man ähnliches auch bei dem sog. schwarzen Tode beobachtet hat ${ }^{36}$, in einer meisterhaften und kaum etwas zu wünschen übrig lassenden, aus den interessantesten und schriftlichen Nachrichten und Chroniken geschöpften Darstellung merkwürdig hervor. Ob wir mit dieser Auffassung des Stichs übrigens mit dem würdigen Hrn. Verf. in Einklang stehen, geben wir demselben zur Beurtheilung anheim: wir sind jedoch sehr geneigt anzunehmen, daß die gesammte Krankheitsconstitution dieser Jahre ein einziges zusammenhängen- 
des, in der eigentlichen Pest ihren Mittelpunkt habendes Wesen darbietet, wovon die katarrhalische und typhöse Constitution so wie der Stich selbst, nur eine einzelne Formation bildete.» ${ }^{37}$

Im gleichen Band des (Janus〉 enthalten ist auch ein Aufsatz des Marburger Professors Karl Friedrich Heusinger (1792-1883), in dem dieser Rückschau hält auf «Die allgemeine erysipelatose Krankheits-Constitution 1830 bis 1847, in ihrer Akme 1838 bis 1844, und die daraus hervorgegangenen Epidemien in Europa und Amerika». Er beschuldigt seine Vorgänger und Kollegen zu großer und zu freier Phantasie, mit der sie die Familie der Erysipelatosen oder Erysipelaceen geschaffen hätten, in deren Umfeld er auch den 〈Alpenstich〉 eingeordnet haben möchte. Dennoch ist auch ihm Guggenbühls Darstellung nicht ganz geheuer, wie folgenden Zeilen zu entnehmen ist: "Der Rothlauf der Lungen war den Alten eine ausgemachte Sache ${ }^{38}$, Forestus erkannte ihn an, wie in neuern Zeiten Peter Frank. In den neuesten Zeiten hat sich Guggenbühler [sic!] bemüht, eine Form der Pleuropneumonie, den (wohl mit Unrecht sogenannten) [!] Alpenstich, eine Krankheit, die offenbar eben so in Italien, Brasilien, Mexiko u.s.w. sehr häufg ist, als Erysipelas pulmonum darzustellen und zwar mit gewichtigen Gründen, für und gegen welche freilich die nahe Verwandtschaft der Anthrakoiden mit den Erysipelatosen geltend gemacht werden kann.» ${ }^{39}$

So lauteten die widersprüchlichen Meinungen und Standpunkte zum «Alpenstich〉 im Jahr 1848.

Hermann Lebert (1813-1878) kam 1854 in einem Vortrag über Conrad Gessner auch auf den sogenannten Stich von 1564 und Guggenbühls Theorie zu sprechen. Er wies eindringlich darauf hin, daß die Bezeichnung (Stich〉 in älteren Texten nur eine Krankheitserscheinung andeutet, keineswegs jedoch deren Natur erklärt. Ebensowenig beschränke sich die Epidemie auf das Hochgebirge. Zwar bestreitet Lebert unter dem Einfluß Schoenleins nicht die Bedeutung ansteckender Brust- und Lungenentzündungen für den Alpenraum, doch gibt er zu bedenken: «Unsere Meinung ist nämlich, daß jene ganze Krankheit eine Brustfellentzündung (Pleuritis), bösartig, unter dem Einflusse des pestartigen Charakters der damaligen Krankheiten gewesen sei, und so zu einer der vielfachen Formen gehöre, unter welchen zur Zeit der herrschenden Pest andere Krankheiten auftreten können. [...] Es ist eine eigenthümliche Tendenz der neueren Krankheitslehre, sich zu ausschließlich mit dem örtlichen Krankheitsprozesse zu beschäftigen. Wir müssen jedoch in dieser, wie in mancher andern Hinsicht, insofern uns den ältern Aerzten wieder nähern, daß wir den allgemeinen Charakter der herrschenden Krankheiten und 
der Epidemien niemals verkennen, und danach die örtlichen Erscheinungen mit der gehörigen Allseitigkeit und Umsicht beurtheilen.» ${ }^{40}$

Wie schon Meyer-Ahrens nähert sich also auch Lebert dem Konzept der Pathokoinose.

Auf der Grundlage früherer Untersuchungen zu den 〈typhösen〉 Krankheiten bezieht August Hirsch (1817-1894) im Jahr 1862 detailliert und umfassend Stellung zur geographischen Verbreitung und historischen Ausdehnung von Pneumonie und Pleuritis. Im besonderen beschäftigt er sich mit den Lungen- und Brustfellentzündungen, «welche in epidemischer Verbreitung auftreten, in der neuesten Zeit übrigens weit seltener, als früher beobachtet, und von den Aerzten vergangener Jahrhunderte unter dem Namen der biliösen oder fauligten (putriden) Pleuropneumonien, neuerdings als Typhoide Pleuropneumonie beschrieben worden sind». ${ }^{41}$ Sie stehen hier jedoch in einem viel weiter aufgefächerten geographischen und literarischen Bezugssystem mit Erstreckung bis ins Mittelalter bzw. über Europa hinaus bis nach Südamerika ${ }^{42}$, doch ist noch allein die Symptomatik entscheidend, keineswegs die exakte Ätiologie. Dafür ist die Lehre von den Krankheitskonstitutionen, von dem (genius epidemicus` noch zu mächtig. Nichtsdestoweniger beginnt bei Hirsch der Einfluß statistischer Methoden sichtbar zu werden. Für die nüchterne Betrachtungsweise spricht gerade der betreffende Abschnitt in der 1886 erschienenen zweiten Auflage der «Historisch-geographischen Pathologies, wo er Klarheit in die Begriffsverwirrung zu bringen sucht: «Schon die Aerzte der letztvergangenen Jahrhunderte hatten sehr richtig erkannt [...], dass die Krankheit - anatomisch betrachtet - dem Begriffe einer fibrinösen Pneumonie entsprach; die Krankheit wurde daher zu den (pestartigen), d.h. bösartig und epidemisch verlaufenden Leiden gezählt, man beschrieb dieselbe als eine 〈giftige〉 oder 〈faulige〉 Lungenentzündung, womit eben der von der gewöhnlichen Pneumonie verschiedene, specifische Charakter der Krankheit angedeutet werden sollte. Später [...] wurde jene Krankheitsform [...] unter dem Namen der ‘typhösen Lungenentzündung〉 beschrieben, [...] oder endlich man sprach, um jedes Präjudiz zu vermeiden, von einer <adynamischen, primär-asthenischen〉 u.s.w. Lungenentzündung.» Hinsichtlich der Pathogenese steht für Hirsch außer Zweifel, daß epidemische Formen von Lungenentzündung existierten ${ }^{43}$.

1866 erscheint «Der Alpenstich in der Schweiz. Ein Beitrag zur Geschichte der Volkskrankheiten> bei Wilhelm Braumüller in Wien. Autor dieser Monographie ist August Feierabend" ${ }^{44}$,Arzt und Sanitätsrath in 
Luzern», dessen Arbeit jedoch - in gefährlicher Nähe zum Plagiat - die ältere von Guggenbühl weitestgehend übernimmt, bis hin zu identischen Formulierungen, der allerdings mit einem grundsätzlich klareren und überschaubareren Duktus als sein Vorbild schreibt. Anmerkungen oder etwa genaue Quellenangaben fehlen bei ihm durchwegs! Die wichtigsten Gesichtspunkte zu Feierabends Darstellung sind: Der «Alpenstich〉 ist für ihn eine 〈rothlaufartige Lungenentzündung bzw. ein innerer «Lungenrothlauf». Die Entstehung der Seuche schreibt er den sogenannten «Schlappwintern» zu, die sich durch Schneearmut, milde Witterung und starken Föhn auszeichneten. Derartige klimatische Verhältnisse sollen das Aufkommen von Rotlauferkrankungen begünstigen, die sich dann zum (Alpenstich` als Volkskrankheit ausweiten würden. Feierabend gibt sich im übrigen deutlich als geistiger Schüler des Tübinger Professors Johann Heinrich Ferdinand von Autenrieth und des nicht minder berühmten Johann Lucas Schoenlein zu erkennen. Auf der anderen Seite befürwortet der Autor ferner wärmstens homoeopathische Heilmethoden sowie die diätetischen Vorschriften Troxlers. Interessant sind die von ihm eingebrachten zwei ausführlichen Krankengeschichten. Vor allen Dingen ist auch seine folgende Feststellung wichtig: «Seit 1857 ist keine Alpenstich-Epidemie in der Schweiz mehr aufgetreten.» ${ }^{45}$

Johann Hermann Baas (1838-1909) streift 1876 nur am Rande die Epidemien «typhöser Pleuropneumonieen» wie sie 1550 und 1564 im Alpengebiet - als Fleckfieber-Varianten [!] - und dann «in der Schweiz, ihrem zweiten Lieblingslande 1652 (Glarus), 1685 (am Genfer See) und 1694-95 in mehr allgemeiner Verbreitung» aufgetreten seien. Für das 18. Jahrhundert gibt er an: «Auch epidemische (biliöse, putride Pleuro-)Pneumonieen herrschten vielfach über die ganze Erde vertheilt, besonders im Frühjahr und Winter. So in Frankreich, der Schweiz, Italien und Deutschland.» ${ }^{46}$ Vom «Alpenstich> keine Spur mehr!

Die Reprise von Heinrich Haeser, dessen «Geschichte der Medicin» 1882 in dritter Auflage erscheint, bringt außer einer immer genaueren Auffächerung der Seuchen, teilweise erheblich eingeschränkt durch die unklaren Termini von Faul-, Schleimfiebern usf. ${ }^{47}$, für den 〈Alpenstich〉 nichts Neues. Der Feierabendsche Hinweis auf das Wissen der Antike wird unterstützt mit der Erwähnung eines gewissen Orlandi, der «die 〈biliöse Pleuritis〉 des Winters 1778/79 zu Rom als eine durchaus mit dem Hippokratischen 〈Erysipelas der Lungen` übereinstimmende Krankheit» ${ }^{48}$ erachtete.

Ernst Leberecht Wagner (1829-1888), dessen Typhusstudie von Quellenkenntnis und kritischer Trennschärfe zeugt, mag sich hinsichtlich des 
〈Alpenstichs〉 nicht festlegen: «Manche Krankheitsfälle, welche von den Einen zur Pneumonie, von den Anderen zum Typhus gerechnet werden, lassen sich nach den Beschreibungen nicht beurtheilen und stellen vielleicht weder das Eine, noch das Andere, sondern eine dritte unbekannte Affection dar. Hierzu gehört wohl die sog. Pneumonia pestilens oder maligna des 16. Jahrhunderts, der Alpenstich (Guggenbühl), die eigenthümliche Krankheit Nordamerikas in diesem Jahrhundert.» ${ }^{49}$

Aus der Frühzeit Überliefertes benützt Max Höfler (1848-1915) aus Bad Tölz, den die nachlassende allgemeine Rezeption nicht beirrt, den «Alpenstich» mit dem erklärenden Synonym «Pneumonia asthenica (typhosa) epidemica» in sein monumentales pathologisches Lexikon von $1899^{50}$ aufzunehmen. Auch die Begriffe «böser Stich», «fauliger Stich», «heimlicher Stich», «Seitenstich» mit allen denkbaren Varianten ${ }^{51}$ fehlen keineswegs. Von hohem Interesse ist insbesondere Höflers etymologische Deutung: «Der Name (Alpstich), aus dem Volksmunde übernommen, kann nur zum dämonistischen ‘ Alp〉 Bezug haben, da die geologische [recte: geographische] Nosologie (1771 Alpenstich) im Volke selbst auch heute nicht zu finden ist. Die ärztliche Schule (in der Schweiz) änderte wohl den volksüblichen Namen Alpstich in (Alpenstich) um; er ist ausserdem nicht einmal an das alpine Hochland der Schweiz gebunden [...]. Der Alpstich ist schon als stechedo ein uralter Krankheitsnamen.» ${ }^{52}$

Als unmittelbare Fortsetzung schließt sich ein Lexikon-Artikel zur «Lungenentzündung» aus der Feder von Karl Sudhoff (1853-1938) an: «Der heftige stechedo war von einem Dämon, einem bösen Alp oder Elf veranlaßt, der dem Kranken seinen Leidensspeer heftig in die Seite gestochen ${ }^{53}$. Noch bis in die Neuzeit hinein hieß die Lungenentzündung (pneumonia) und ihre noch nicht von ihr getrennte Zwillingsschwester, die Brustfellentzündung (die pleuritis oder pleuresia), der Alpstich.» ${ }^{54}$

Ob Guggenbühl bei seiner Namensprägung auf den quälenden Alp Bezug nahm oder ob er damit auf die Ursprungslandschaft der Seuche hinweisen wollte oder beides, kann nur noch spekulativ entschieden werden.

Sieben Tage gab man dem Kranken zu leben, wenn er vom (Alpenstich) ergriffen wurde. Auf gut siebeneinhalb Jahrzehnte bemessen war die Lebenszeit des «Alpenstichs» selber, wenn man darunter nur den pathologischen Begriff verstehen möchte ${ }^{55}$. Als Vertreiber des Phantoms von der wie die Pest gefürchteten Epidemie machte sich ein Mann verdient, der in die Annalen der Heilkunst weniger als Arzt, denn als Historiker Eingang gefunden hat: Georg Sticker. Georg Sticker, geboren 1860 in Köln, studierte 
in Straßburg und Bonn, habilitierte sich 1895 bei Riegel in Gießen, nachdem er jahrelang seine Praxis in Köln geführt hatte. 1897 Mitglied der Deutschen Kommission zur Erforschung der Pest in Indien, wurde Sticker 1898 zum Extraordinarius für Innere Medizin ernannt. Nach Riegels Tod kehrte er der Universität den Rücken, wirkte lange Jahre als praktischer Arzt und Internist in Köln und Bonn sowie als Oberarzt und Lazarettdirektor in Münster, wo er 1920 zum Honorarprofessor bestellt wurde, und ging dann 1921 an die Bayerische Julius-Maximilians-Universität nach Würzburg. Dort richtete er als Ordinarius ein erstes medizinhistorisches Institut ein. 1934 wurde Sticker aus politischen Gründen entlassen. Er starb hochbetagt und hochgerühmt $1960 \mathrm{im} \mathrm{101.} \mathrm{Lebensjahr} \mathrm{in} \mathrm{Zell} \mathrm{am} \mathrm{Main.}$

Sticker mußte zwangsläufig früher oder später auf den (Alpenstich) stoßen. Zwar hatte er 1884 in Bonn ein anatomisch-osteologisches Thema für seine Dissertation ${ }^{56}$ gewählt und in seiner klinischen Tätigkeit als Internist vorwiegend Probleme der Verdauung untersucht ${ }^{57}$, selten dabei jedoch historische Gesichtspunkte außer Acht gelassen. Seit Beginn seiner bevorzugten Forschungstätigkeit hat sich Sticker dem Studium der Infektionskrankheiten verschrieben. Es entstanden in seiner frühen Schaffensphase Arbeiten zur Tuberkulose ${ }^{58}$, zum Keuchhusten ${ }^{59}$, zu Lepra und Pest $^{60}$, zum akuten Erythem ${ }^{61}$ usf.

Zum (Alpenstich) äußert sich Sticker erstmals, als er 1902 in einer Monographie «Die Entwicklung der ärztlichen Kunst in der Behandlung der hitzigen Lungenentzündungen» skizziert. In einer Anmerkung zum Abschnitt 〈Pestpneumonie〉 heißt es dort: «Ueber die merkwürdigen Epidemien der Pneumonia alpina, des bösartigen Stiches, des Alpenstiches, dürfte eine eingehende Untersuchung von Werth sein. So viel ich sehe, handelt es sich bei den älteren Epidemien, welche Conrad Gesner, Thaddäus Dunus, Haller beschreiben, um Pestpneumonien, wofür wenigstens dieses spricht, dass die Epidemie des Dunus im Jahre 1564 zwischen zwei Beulenpestseuchen fält, und dass Gesner selbst im Jahre 1565 an der Pest gestorben ist. [...] Dagegen könnten die von Schönlein, Lebert u. A. beobachteten Pneumonieseuchen (in Hospendal und Andermatt 1833 u.s.w.) auch Milzbrandepidemien sein». ${ }^{62}$

Etwa zur gleichen Zeit geistert das Gespenst des 〈Alpenstichs〉 allerdings noch durch die Fachliteratur. Der eigentlich historisch versierte Internist und Neurologe Ernst von Leyden (1832-1910) schreibt 1910 wie folgt: «Von grosser Bedeutung waren die Untersuchungen über das epidemische Auftreten und die Ansteckungsfähigkeit der Pneumonie, welche der bakteriologischen Forschung unmittelbar vorhergingen. Bereits Wunderlich in seiner 
auch heute noch schätzbaren speciellen Pathologie und Therapie spricht von sporadisch und von epidemisch auftretender Pneumonie. Im Frühjahr ist sie häufig, am seltensten im Hochsommer. E. Klebs machte darauf aufmerksam, dass die Pneumonie, welche zur Zeit der Schneeschmelze in der Schweiz auftritt und als Alpenstich bezeichnet wird, durchaus ein epidemisches Verhalten zeigt.» ${ }^{63}$

Georg Sticker hingegen beginnt mit der Auswertung historischer Quellen, um der Sache auf den Grund zu gehen. Aus den im Nachlaß Sticker vorhandenen Lesescheinen der Bibliotheken in Gießen und Straßburg i.E. wird ersichtlich, daß er ab dem Jahr 1901 systematisch alte Literatur auf den (Alpenstich) hin untersucht hat. Zur Ordnung der Epidemiefolgen hat er eine Sammlung von ca. 380 kleinformatigen Blättern angelegt. Die Eintragungen umfassen unter dem Arbeitstitel «Seuchenhafte Lungenentzündungen, chronologisch» hunderte von Seuchenzügen der Jahre und Jahrzehnte zwischen 1348 und 1890. Direkte Verwendung zur Publikation scheint das Verzeichnis nicht gefunden zu haben. Wahrscheinlich war die Kompilation gedacht als Grundlage für die wissenschaftliche Argumentation und Beweisführung in einer Nachfolgeschrift über die infektiöse Pneumonie und den 〈Alpenstich〉 im besonderen. Eine solche Studie ist jedoch nie erschienen, und auch in seiner Darstellung der Pest in den beiden diesbezüglichen Bänden der «Abhandlungen aus der Seuchengeschichte und Seuchenlehre` bezieht er sich nicht auf den 〈Stich〉.

Im Jahr 1913 hat sich die zwölf Jahre zuvor ausgesprochene Hypothese zum 〈Alpenstich〉 so weit bestätigt und verdichtet, daß Sticker auf dem vom 6 . bis 12 . August d. J. in London stattfindenden «17th International Congress of Medicine» vor den Teilnehmern der Section 23 - «History of Medicine» - ein Referat hält zum Thema: «Die Geschichte des Alpenstichs».

Seine darin ausgesprochene Kritik an Guggenbühl ist so vernichtend, daß die Lehre vom (Alpenstich) damit als abgeschlossen gelten kann.

Angesichts der herausragenden Bedeutung des Stickerschen Beitrags und seiner schweren Zugänglichkeit ${ }^{64}$ sei - trotz gelegentlicher Wiederholung zu oben bereits Dargestelltem - hier als Synthese und Quintessenz der Aufsatz Stickers im Wortlaut des endgültigen Manuskripts wiedergegeben: ${ }^{65}$ 


\title{
Die Geschichte des Alpenstichs
}

\author{
Von Professor Georg Sticker, Münster i.W.
}

In den schweizer Bergen tritt seit Jahrhunderten von Zeit zu Zeit eine bösartige Lungenentzündung epidemisch auf. Das Volk erinnert sich solcher Ausbrüche mit den Namen des heimlichen Stiches, des faulen Stiches, des bösartigen Stiches, im Gegensatz zum Stich schlechthin, der die gewöhnliche Lungenentzündung bedeutet. Der Name würde wol über die Grenzen seines Vaterlandes nicht hinausgedrungen sein, wenn nicht ein großer Lehrer ihn in die Klinik und Epidemiologie eingeführt hätte. Lukas Schönlein in Zürich beobachtete das Uebel in den Jahren 1834 und 1835 in den Urkantonen. Den Ausbruch im Spätherbst 1834 zu Ursern deutete er als larvirtes Wechselfieber, weil er ihn den Wasserläufen entlang verfolgen konnte. Von dieser Auffassung kam er zurück, als er dasselbe Uebel zu Beggenried und Buochs im Frühjahr 1835 aufs neue studirte und es diesmal mit einer ausgesprochenen Rotlaufkonstitution einhergehen sah. ${ }^{66}$ Vielleicht ist seine Deutung im zweiten Falle ebenso anfechtbar wie im ersten. Gewiss aber ist, dass der grosse Seuchenkenner auf dem richtigen Wege war, im Gegensatz zu seinen Schülern, die später unter dem Namen des Alpenstiches eine besondere Seuche konstruirten. Diese Seuche soll seit Jahrhunderten ihre ausschliessliche Heimat in den schweizer Hochtälern haben, dort fast jedes Jahr vereinzelte Opfer fordern und nach besonders milden Wintern zur Zeit der Schneeschmelze epidemische Macht gewinnen, dann die Alpentäler veröden und sogar über die Grenzen des Berner Hochlandes, des Wallis und Graubündens hinaus in das Rheinland, Waadtland, Tessin, Veltlin hinabsteigen und weiter bis zu den nördlichen und südlichen Meeresküsten mit dem menschlichen Verkehr vordringen.

Diese Vorstellung, die zuerst Guggenbühl im Jahr 1838 zu begründen versucht und dann Feierabend im Jahre 1866 übernommen hat, ist unhaltbar. Weder gibt es eine einheitliche Ursache des bösartigen Alpenstiches noch hat sich diese Krankheit je wandernd als eine weite und breite Epidemie über die Grenzen der Schweiz ausgedehnt. Die bestechende Darstellung Guggenbühls beruht auf einer irrigen Auslegung der Quellen über vergangene Epidemien und auf einer willkürlichen Aneinanderreihung von gleichzeitigen und aufeinander folgenden Ereignissen zu einer ursächlich zusammenhängenden Kette. Wie wenig Guggenbühl befähigt war, historische Daten für epidemiologische Aufgaben zu verwerten, hat für seine 
Darstellung der Stichepidemien des Jahres 1564-65 Meyer-Ahrens in einer bedeutenden Schrift vom Jahre 1848 gezeigt. Nach dieser Schrift und ihren Quellen bei Conrad Gesner, Thaddaeus Dunus, Johann Weyer u.s.w. schrumpft der grosse Alpenstich vom Jahre 1564 zu einer kleinen Nebenerscheinung der damaligen Pestkonstitution zusammen. In Wirklichkeit war er, wie ich dann weiter nachweisen konnte, nichts anderes als die uns heute wohlbekannte Winterform der Beulenpest, die pestige Lungenentzündung, wie sie sich seit der Zeit des Schwarzen Todes bis in unsere Tage in zahlreichen Pestläufen gezeigt hat. ${ }^{67}$

Was für die Ausbrüche des bösen Stiches im Jahre 1564 gilt, gilt auch für die weiteren, deren Beschreibungen durch Haller, Tissot, Lebert u.s.w. in die allgemeine Fachliteratur übergegangen sind oder sich aus Schweizer Schriften und Archiven sammeln lassen; und es gilt für die epidemische Lungenentzündung überhaupt. Es handelt sich bei ihr nicht um eine selbständige Seuche, sondern um eine Teilerscheinung verbreiteter Volkskrankheiten, die nur unter ganz bestimmten Bedingungen hervortritt. ${ }^{68}$

Aus mehr als hundert Pneumonieepidemien ausserhalb der Schweiz und aus ihrer Vergleichung mit den schweizerischen Alpenstichepidemien geht hervor, dass ein grundsätzlicher Unterschied zwischen diesen und jenen nicht besteht. Das klinische Bild oder vielmehr die verschiedenen klinischen Bilder des bösartigen Stiches, die die Schweizer Epidemiologie kennt, finden wir in allen Ländern Europas, besonders in den Rheinlanden, in Baden, in Bayern, in Oesterreich, auffallend häufig in Italien.

Der sogenannte Alpenstich und die ihm gleichen Epidemien in Europa haben zu verschiedenen Zeiten verschiedene Ursache und entsprechend verschiedene klinische Form und Verlauf. Das eine Mal handelt es sich um Pestpneumonie ${ }^{69}$, das andere Mal um die Milzbrandpneumonie, das dritte Mal um die Erysipelpneumonie, das vierte Mal um die Influenzapneumonie, in einer fünften grossen Reihe von Ausbrüchen um den Pneumotyphus, in jüngeren kleinen Epidemien um die Papageienpneumonie, dann und wann vielleicht auch um Malariapneumonien. ${ }^{70}$

Aber alle diesen grossen und kleinen Ausbrüche von maligner, asthenischer, biliöser, typhöser Lungenentzündung haben ausser ihrer Gefährlichkeit das Gemeinsame, dass sie sich in der kalten und nasskalten Jahreszeit ereignen, zu Anfang oder im Verlauf des Winters, und daß sie mit dem Eintreten eines entschiedenen Frühlingswetters rasch nachlassen und ferner, dass sie kaum jemals für sich allein dastehen - wenn man von den Psittacosisausbrüchen absehen will - sondern sich an eine vorherbestehende 
Seuche, an einen gewöhnlichen Pestgang, einen gewöhnlichen Typhusausbruch u.s.w. anschliessen oder vielmehr daraus oder aus einer stehenden Krankheitskonstitution, wie der erysipelatösen, der Malariaendemie, der Anthraxendemie u.s.w. hervorgehen. Eine Pestseuche, die im Sommer als Bubonenpest gewütet hat und im Herbst zu Ende ging, erhebt sich gelegentlich im Winter aufs neue als Lungenpest; ein Bauchtyphus, der im Herbst und Winter das gewöhnliche Bild zeigte, wandelt sich plötzlich gegen Wintersende bei herrschender Tramontana oder beim Wehen des Föhns in einen epidemischen Pneumotyphus um; wo vorher Gesichtsrose, Puerperalfieber, Wundsepsis, Hospitalinfektionen sich häuften, wo eine Milzbrandherrschaft sich im fliegenden Zungenkrebs oder in der schwarzen Pustel bei Vieh und Menschen äusserte, da kann es im Winter zu epidemischer Lungenentzündung mit den Zeichen der spezifischen Infektion kommen.

$\mathrm{Zu}$ einer bedeutenden Wanderseuche hat sich kein derartiger Ausbruch erhoben, weder in der Schweiz noch ausserhalb der Schweiz. Ihr gelegentliches Aufkommen an verschiedenen Orten zugleich unter der Herrschaft der gleichen Witterungsverhältnisse bedingt hier und da den Schein der Wanderepidemie. Aber in Wirklichkeit handelt es sich um beschränkte stehende Ortsausbrüche von begrenzter Zeitdauer und mit geringer Neigung, auf Menschenansiedlungen in der näheren Umgebung überzugehen.

Die Winterform der Pest, des Typhus, der Milzbrandseuche u.s.w. in Gestalt epidemischer Pneumonie rechtfertigt die alten Begriffe der Constitutio epidemica und Constitutio annua. ${ }^{71}$ Man kann diese Wörter entbehren, aber die Tatsachen, die ihnen zugrunde liegen, verdienen in der Epidemiologie grössere Rücksicht, als sie bisher gefunden haben. Zahlreiche Irrtümer in der Seuchendiagnose beruhen auf ihrer Vernachlässigung, wie der Verfasser in einem besonderen Buche über die epidemische Lungenentzündung zeigen wird.

So weit Stickers Vortrag. Im gleichen Jahr 1913 weist er in einer Rezension ergänzend auf die Abgrenzung zu normalen Influenza-Epidemien hin; an diesen sterbe nämlich so gut wie kein Mensch. "Als tödliche wandernde Katarrhseuchen sind Winterpest, Wintertyphus, Lungenerysipel, Diplokokkenpneumonien bekannt. Sie zogen im 16.Jahrhundert und weiterhin als ¿Alpenstich) wiederholt aus der Schweiz rheinabwärts.» ${ }^{72}$

Damit endet die historische Beschreibung des 〈Alpenstichs〉 im 19. und frühen 20. Jahrhundert. 
Wie wenig die zeitgenössische Wissenschaft sich um die historische Forschung kümmert, beweist folgendes Zitat, eine Begriffsdefinition unter dem Stichwort 〈Alpenstich〉: «epid[emische] Pneumonie in bestimmten (Schweizer) Alpentälern ».73 Ein Blick in die Geschichte schadet der Praxis beileibe nicht!

Die in der Lehre vom «Alpenstich) tangierten Syndrome der Lungen und der Pleura bleiben in ihrer geographischen, historischen und epidemiologischen Entwicklung und Bekämpfung noch heute ein Arbeitsfeld, das zur Quellenforschung und -deutung herausfordert. Mangels kritischer, ausgreifender Untersuchungen sind z. B. weiterreichende Thesen und Spekulationen zum Erregerspektrum der Krankheit hier noch nicht am Platze. Es sollte nicht verwundern, wenn man auf den Spuren des sogenannten Alpenstichs zu Ergebnissen gelangte, die über den Ansatz von Georg Sticker hinausreichen. Ähnliches geschah unter anderem bereits in der Frage der in Europa vorherrschenden Infektkette bei der Pest und den sich daraus ergebenden Konsequenzen ${ }^{74}$ sowie bei der Klärung der Ätiologie der Lepra.

Unvermindert gültig bleiben wird jedoch Stickers Fazit: «Weder die Vorstellung von einer einheitl[ichen] Ursache des Alpenstiches ist haltbar, noch die Vorstellung, dass der Alpenstich je als eine bedeutende Wanderseuche sich verhalten hat.» ${ }^{75}$

\section{Anmerkungen}

1 Zum Ursprung dieser Idee ef. Crombie, Alastair Cameron: Robert Grosseteste and the Origins of Experimental Science 1100-1700. 2nd impr. Oxford: Clarendon, 1962. pp. 61-90.

2 Zur Forderung an den $\mathrm{W}$ issenschafter nach in sich widerspruchsfreier Abdeckung aller Gesichtspunkte v. Mittelstraß, Jürgen: Die Rettung der Phänomene: Ursprung und Geschichte eines antiken Forschungsprinzips. Berlin: de Gruyter, 1962.

3 Dies gilt insbesondere für die einzelnen Fieberarten, deren Bezeichnungen allein bei Galen schon die Zahl von 70 weit überschreiten. Bzgl. pneumologischer Aspekte cf. Altmann, Gustav: Die wichtigsten Kurmethoden der Pneumonie in ihrem Zusammenhang mit den jeweils über das Wesen der Pneumonie herrschenden medicinischen Anschauungen. Straßburg: Fischbach, 1887. (Straßburg i.E., Univ., Med. Fac., Diss., 1887).

4 Das 18. Jh. gilt auch als das Jahrhundert der Systeme, was sich z. B. bei Philippe Pinel (1775-1826) deutlich manifestiert.

5 Eindrucksvolle Zeugnisse für dieses Phänomen geben bsp. die ebenso grandiose wie falsche Humoralpathologie, die angebliche Kenntnis vom Blutkreislauf seit der Antike, die Interpretationen biblischer Dermatosen. Wirkungsmächtige Fehlschlüsse waren 
besonders im Bereich der Embryologie verbreitet, cf.: Reisert, Robert: Der siebenkammerige Uterus: Studien zur mittelalterlichen Wirkungsgeschichte und Entfaltung eines embryologischen Gebärmuttermodells. Pattensen/Han.: Wellm, 1986. (Würzburger medizinhistorische Forschungen; 39) [Würzburg, Univ., Med. Fak., Diss., 1986] sowie Hippéli, Roman, u. Keil, Gundolf: Zehn Monde Menschwerdung. Biberach an der Riss: Basotherm, 1982.

6 «Zur Legendenbildung in der Medizin» v. Thies, Hans-Jürgen: Der Diabetestraktat ${ }^{\mathrm{c}} \mathrm{Abd}$ al-Lațif al-Bağdādī's: Untersuchungen zur Geschichte des Krankheitsbildes in der arabischen Medizin. Bonn: Orientalisches Seminar der Universität, 1971. (Bonner Orientalistische Studien; 21).

7 Mit bewundernswerter Konsequenz tat dies z. B. der Amerikaner Abe Lilienfeld, ef. Hannaway, Caroline: Abraham M.Lilienfeld (1920-1984). in: Bulletin of the History of Medicine 58 (1984): 558-559.

8 cf. Sticker, Georg: Die Bedeutung der Geschichte der Epidemien für die heutige Epidemiologie: Ein Beitrag zur Beurteilung des Reichsseuchengesetzes. Gießen: Topelmann, 1910. (Zur historischen Biologie der Krankheitserreger; 2) u.v.a. Heutzutage mehr als Medizinhistoriker bekannt, fühlte sich Sticker doch zeitlebens als Arzt. Der Terminus «Stickersches Syndrom» (Erythema infectiosum acutum) erinnert an das jahrzehntelange segensreiche Wirken Stickers in Klinik und freier Praxis.

9 Ein Blick in die Bibliographie Stickers läßt seine Vielseitigkeit und Interessensbreite ahnen, allerdings nur die Spitze des wissenschaftlichen Eisbergs, dessen wahre Ausmaße die Durchsicht und Aufarbeitung von Stickers Nachlaß sichtbar machen wird; cf. Lossen, Heinz: Ö̈. Prof. (emer.) Dr.med.Georg Sticker, Ordinarius für Geschichte der Medizin an der Julius-Maximilians-Universität, Würzburg, zum 100.Geburtstag. Darmstadt: Darmstädter Echo, 1960 (leider unvollständig!).

10 1. Aufl. (1857). p. 565; cf. 5. Aufl. (1893). p. 425.

11 Johann Jakob Guggenbühl (1816-1863) eröffnete schon vier Jahre nach seiner Promotion, auf dem Abendberg bei Interlaken eine Heilanstalt für Kretinen (1841); die Erforschung des Kretinismus, die praktische Sorge und medizinische wie pädagogische Betreuung der Patienten, die Verbreitung seiner philanthropischen Ansichten über die Pflege und Heilung geistig Kranker bestimmten fortan sein Leben, das nur 47 Jahre währen sollte, aber wegweisend war. cf. Kanner, Leo: Johann Jakob Guggenbühl and the Abendberg. in: Bulletin of the History of Medicine 33 (1959): 489-502; Rengger, Hermann: Dr.med.J.Guggenbühl, der Begründer der ersten Heilanstalt für Kretinen, und seine Anschauungen über den Kretinismus. Zürich, Univ., Med. Fak., Diss., 1927; Balmer, Heinz: Dr.med.J.J.Guggenbühl (1816-1863). in: Neue Deutsche Biographie (NDB). Berlin: Duncker \& Humblot. vol. 7 (1966). pp. 294-295. [Beide letztgenannten Autoren halten Guggenbühls Beschäftigung mit dem 〈Alpenstich〉 keiner Erwähnung für würdig!]

12 v. Guggenbühl, Johann Jakob: Der Alpenstich endemisch im Hochgebirg der Schweiz und seine Verbreitungen/m. Vorw. v. Prof.Troxler. Zürich: Höhr, 1838. p. VIII. - Zur Medizintheorie Troxlers v. Heusser, Peter: Der Schweizer Arzt und Philosoph Ignaz Paul Vital Troxler (1780-1866). Seine Philosophie, Anthropologie und Medizintheorie. Basel: Schwabe, 1984. (Baseler Veröffentlichungen zur Geschichte der Medizin und der Biologie; 34) [Basel, Univ., Med. Fak., Diss., 1983].

13 Hierzu als fundierte Darstellung: (Koelbing-)Waldis, Vera: Der «Stich〉 von 1564 - eine primäre Lungenpest. in: Gesnerus 40 (1983): 223-228. Ein weiterer Hinweis bei: Fischer, 
Hans: Conrad Gessner (26. März 1516-13. Dezember 1565): Leben und Werk. Zürich: Leemann, 1966. (Neujahrsblatt 168 (1966) [hrsg. v.d. Naturf. Ges. in Zürich] = Vierteljahrsschrift der Naturforschenden Gesellschaft in Zürich 110 (1965), Beiheft). p. 130. Fischers Hypothese vom (Alpenstich) als einer Viruskrankheit ist zumindest für die Epidemie von 1564 auszuschließen, ebenso Salzmanns Deutung als bösartigem grippalen Infekt. Interessanterweise findet sich die Virus-These auch später noch, cf. Büchi, Jakob: Conrad Gessner (1516-1565) als Zürcher Stadtarzt. in: Festschrift für A. Lutz und J.Büchi $[\ldots] /$ hrsg. v. Hans-Rudolf Fehlmann u. François Ledermann. Zürich: Juris, 1983. (Veröffentlichungen der Schweizerischen Gesellschaft für Geschichte der Pharmazie; 2). pp.113-138, hier p. 133; sowie Id.: Die Entwicklung der Rezept- und Arzneibuchliteratur. 2. Teil: Die Autoren, ihre Werke und die Fortschritte im 16. Jahrhundert. Zürich: Juris, 1984. (Veröff. d. Schweiz. Ges. f. Gesch. d. Pharm.; 4). p.127.

14 Franz Richli (?-?) aus Malters, Dr. med., Sanitätsrat des Kantons Luzern, Vizepräsident des Sanitätsrates 1821-1831. Unterschiedliche Anschauungen über den (Alpenstich〉 führten 1806 zur Kontroverse zwischen Troxler und Richli und dem Weggang des ersteren nach Wien.

15 v. Guggenbühl. op. cit. p. 70-72, 1.

16 v. Feierabend, August: Der Alpenstich in der Schweiz: Ein Beitrag zur Geschichte der Volkskrankheiten. Wien: Braumüller, 1866. p.3. Dasselbe bestätigt auch Sticker in einem Vortragsmanuskript von 1913.

17 v. Thaddaei Dvni Locarnensis medici, epistolae medicinales [...]. Tigvri: excudebat Ioannes VVolphius, typis Froschouianis. M.D.XCII. f. 132 $-133^{\mathrm{r}}$. cf. Waldis. op. cit. Zur Bewertung Dunos v. Salzmann, Carl: Thaddeo Duno von Locarno als Stadtarzt in Zürich: Eine biographische Studie. in: Vierteljahrsschrift der Naturforschenden Gesellschaft in Zürich 85 (1940): 337-344. Dort heißt es (p. 340): «Zu den bedeutendsten Arbeiten Duno's gehören seine epidemiologischen Aufzeichnungen über die Pleuritis (zur Zeit ihres Auftretens in Zürich 1563 bis 1564 (Stich» oder Alpenstich genannt) [hier dürfte Salzmann ein Anachronismus unterlaufen sein! Anm. d. Verf.], sowie über die Pest, welche zur gleichen Zeit in Zürich so viele Opfer forderte. Sprengel erklärte in seiner Geschichte der Arzneikunde 1821-1837, Duno's Abhandlung über den Aderlass bei Pleuresie sei das Bedeutendste und Gründlichste, was in jenem Zeitalter über diesen Gegenstand geschrieben wurde.»

18 Zur Terminologie v. Janssen, Hubertus Anna: Beitrag zur Kenntniss vom epidemischen Auftreten der Pneumonia biliosa. in: Deutsches Archiv für klinische Medicin 35 (1884): 355-381.

19 v. Feierabend. op. cit. p. 113.

20 Einschränkend meint Guggenbühl. op. cit. pp.93-94: «Eine vollständige Auffassung fehlt aber noch durchgreifends und ist um so weniger bei einer Krankheit zu erwarten, deren grosse Mannigfaltigkeit in der Erscheinungsweise selbst bis zur Stunde der Stein des Anstosses blieb.» [!] - Feierabend (op. cit. p.5) drückt sich recht vorsichtig aus: «Wie indessen auch die Ansichten der Aerzte im Laufe der Zeiten über die Natur und den Charakter der mörderischen Krankheit wechselten, so lässt sich doch aus ihren Aufzeichnungen eine gewisse gleichartige Grundform nicht verkennen.»

21 cf. Guggenbühl. op. cit. p. 74-93. Zur Ähnlichkeit des Krankheitsbildes mit der Pest cf. Sticker, Georg: Abhandlungen aus der Seuchengeschichte und Seuchenlehre. I. Band: Die Pest. 2. Teil: Die Pest als Seuche und als Plage. Gießen: Töpelmann, 1910. p. 256. 
22 Ähnliche Verfahren zur Entfernung der (materia peccans) waren jahrhundertelang bei der Behandlung ansteckender Krankheiten üblich.

23 v. Feierabend. op. cit. p.65. Eigene Berechnungen nach den spärlichen und wenig vertrauenswürdigen Zahlenangaben bei Guggenbühl und Feierabend.

24 cf. Castiglioni, Arturo: Der Aderlaß in der Renaissance; Die Phlebotomie in der Neuzeit. in: Ciba-Zeitschrift 66 [6 (1954)]. hier pp. 2203-2209.

25 v. Abhandlung über das Faulfieber, ein Geschenk für heilende Landärzte, samt einem Anhange einer fäuligten Epidemie im Kanton Bern von Hrn. von Haller, mit Anmerkungen von Hrn. Leibarzt Zimmermann ... Solothurn: Gaßmann, 1786. cf. d. frz. Original: Haller, Albert de: Mémoire sur une maladie épidémique, arrivée dans le Canton de Berne en 1762. in: Histoire de l'Académie Royale des Sciences. Paris 1763. pp. 167-171.

26 v. Guggenbühl. op. cit. p. 90. - Eine knappe «Geschichte der Pneumonie» mit zugehöriger «Geschichtliche[r] Uebersicht der Therapie» gibt: Leyden, Ernst von: Pneumonie. in: Die Deutsche Klinik. Berlin: Urban \& Schwarzenberg. vol.2 (1903). hier pp.251-253, 289-294. Die neuere Entwicklung skizziert: Gsell, Otto: Geschichte der Pneumonie. in: Sudhoffs Archiv 68 (1984): 182-216; Id.: Die Geschichte der Pneumonien - Wandel vom 19. zum 21. Jahrhundert. in: Praxis und Klinik der Pneumologie 38 (1984): 379-388.

27 v. Feierabend. op. cit. p. 65. - Zum historischen Überblick über den Aderlaßstreit v. Looschen, Annette: Die ältere Geschichte des Krankheitsbegriffes Pleuritis. Kiel, Univ., Med. Fak., Diss., 1969.

28 v. Dr. J.L.Schönlein's allgemeine und specielle Pathologie und Therapie. Nach dessen Vorlesungen niedergeschrieben $u$. hrsg. v. einigen seiner Zuhörern. 5., sorgf. verb. u. verm. Aufl. St.Gallen: Litteratur Comptoir, 1841. Theil 1. pp. 203-204. Dort bleibt die Pleuritis eine Krankheit sui generis und wird nicht mit dem «Alpenstich〉 confundiert. Zur Frage der Authentizität der Schönleinschen Äußerungen v. Hirsch, August: Geschichte der medicinischen Wissenschaften in Deutschland. München: Oldenbourg, 1893. (Geschichte der Wissenschaften in Deutschland; 22). pp. 595-596; Sticker, Georg: Entwicklungsgeschichte der medizinischen Fakultät an der Alma Mater Julia. in: Aus der Vergangenheit der Universität Würzburg: Festschrift zum 350jährigen Bestehen der Universität/hrsg. v. Max Buchner. Berlin: Springer, 1932. pp. 578-579.

29 v. Haeser, Heinrich: Historisch-pathologische Untersuchungen. Als Beiträge zur Geschichte der Volkskrankheiten. Dresden: Fleischer. Erster Theil. 1839. Zweiter Theil. 1841.

30 op. cit. I (1839): pp. 265-271. II (1841): pp. 14-15, 20, 94-95, 113-116, 144-146, 433-4.34, 488, 489-492, 515-519.

31 op. cit. I: p. 267. II: p. 113.

32 cf. Haeser, Heinrich: Lehrbuch der Geschichte der Medicin und der Volkskrankheiten. Jena: Mauke, 1845. pp.474-475 bzw. 481-484. - Aus Frankreich berichtet Guillaume de Baillou (1538-1616) über die gefährlichen ‘Pleuritides erysipelatodes, non phlegmonodes) der Jahre 1570-1579.

33 v. Meyer-Ahrens, Konrad: Der Stich in den Jahren 1564 und 1565: im Zusammenhange mit den übrigen Epidemieen der Jahre 1562-1566. Zürich: Schulthess, 1848. pp.64, 69-70, 72-73, 78-79, 90-93.

34. Zum (Alpenstich): Meyer-Ahrens. op. cit. pp.61-93, 109, 156-160.

35 ef. als Biobibliographie: Röthlisberger, Paul: Der Zürcher Arzt Conrad Meyer-Ahrens (1813-1872) - Medizinhistoriker, Epidemiologe und Balneograph. in: Gesnerus 30 
(1973): 122-142. - Fragwürdig erscheint ebenfalls eine Bemerkung Guggenbühls zur Identität von «Rothsucht» und Scharlach, cf. Haeser (1839). op. cit. p. 309.

36 Zum Krankheitsbegriff und -bild der typhösen Pneumonie (z.T. auch als Pneumotyphus oder Pestilenzialtyphus bezeichnet) v. Seitz, Franz: Bemerkungen über die ältere französische Literatur des Pestilenzialtyphus. in: Janus [Breslau] 3 (1848): 444-455; Hirsch, August: Handbuch der historisch-geographischen Pathologie. Erlangen: Enke, 1862-1864. Bd.2. pp. 38-47; ibid. 2. Aufl. 1886. 3. Abth. pp. 109-110; Haeser (1845). op. cit. pp.481-484, 798-800; Janssen. 1.c.; Wagner, Ernst Leberecht: Der sogenannte Pneumotyphus. in: Deutsches Archiv für klinische Medicin 35 (1884): 355-381.

37 v. Henschel, August W.: [Rez. zu:] Zürich bei Friedrich Schulthess 1848. Der Stich in den Jahren 1564 und 1565 im Zusammenhange mit den übrigen Epidemien der Jahre 1562-1566, dargestellt von Dr. Konrad Meyer-Ahrens. S.182. 8. in: Janus [Breslau] 3 (1848): 815-818. - Zum Konzept der Pathokoinose, d.h. zum Versuch, die Krankheitszustände einer bestimmten Epoche zusammenhängend zu betrachten cf. Grmek, Mirko D.: Préliminaires d'une étude historique des maladies. in: Annales: Économies, Sociétés, Civilisations 24 (1969): 1473-1483; Id.: Les maladies à l'aube de la civilisation occidentale. Recherches sur la realité pathologique dans le monde grec préhistorique, archaïque et classique. Paris: Payot, 1983. pp. 14-17.

38 Anspielung auf Teile des Corpus hippocraticum, cf. Vierordt, Hermann: Lungenkrankheiten (ausschliesslich Tuberkulose). in: Handbuch der Geschichte der Medizin/hrsg. v. Max Neuburger u. Julius Pagel. Jena: Fischer, 1903. Bd.2. pp.615-616. Zum «Erysipèle dans le poumons v. Euvres complètes d'Hippocrate/Trad. nouv. avec le texte grec en regard par Émile Littré. Réimpr. de l'Edition Paris 1851. Amsterdam: Hakkert, 1979. T. 7: Des maladies, Livre troisième: Des affections internes [...]. pp. 84-89, 180-187 [dt. in: Die Werke des Hippokrates: Die hippokratische Schriftensammlung in neuer deutscher Übersetzung/hrsg. v. Richard Kapferer unter Mitw. v. Georg Sticker. Stuttgart: Hippokrates, (1933-1940). Bd.3, T.XVIII. pp.55-56, 67-69. Bd.4, T.XIX. pp. 33-34]. - Zur Peripleumonie speziell v. Preiser, Gert: Peripleumonie in den Schriften der knidischen Ärzteschule. in: Medizingeschichte in unserer Zeit: Festgabe f. Edith Heischkel-Artelt u. Walter Artelt z. 65. Geburtstag/hrsg. v. Hans-Heinz Eulner. Stuttgart: Enke, 1971. pp.31-35. - Zu den inneren Anthraxformen v. Jahn, Ferdinand: Beiträge zur Geschichte der Carbunkel-Krankheiten mit Ausschluß der Pest. in: Janus [Breslau] 1 (1846): 369-414, 485-550.

39 v. Heusinger, Carl Friedrich: Die allgemeine erysipelatose Krankheits-Constitution 1830 bis 1847, in ihrer Akme 1838 bis 1844, und die daraus hervorgegangenen Epidemien in Europa und Amerika. in: Janus [Breslau] 3 (1848): 154-165, 525-546, 744-793, hier pp. 161-162.

40 cf. Lebert, Hermann: Conrad Gesner als Arzt. Zürich: Bürkli, 1854. pp. 28-33.

41 v. Hirsch (1862). op. cit. pp. 20-49 sowie 40-45. Aus dem Zeitraum von 1521 bis 1858 greift Hirsch hier 186 Seuchenzüge bzw. -jahre, wovon 21 auf die Schweiz entfallen (zum Vergleich: Deutschland: 20; Italien: 35; Frankreich: 60), wobei als Quelle keineswegs nur Guggenbühl benutzt wird. - Zur Geschichte der Pleuritis v. Looschen. l.c.; Baffoni, Aroldo: Storia delle pleuriti da Ippocrate a Laennec/con prefazione di Adalberto Pazzini. Roma: Sograro, 1947.

42 Am Rande erwähnt sei der Hinweis, «dass der primäre Pneumotyphus auf der Küste von 
Peru, dasselbst unter dem Namen tabardillo y costado (d.h. typhöser Lungenstich) bekannt, die häufigste dort vorkommende Typhusform ist.» ef. Hirsch (1862). op. cit. p. 46.

43 v. Hirsch (1886). op. cit. pp. 104-105. - Zur Namensgebung, unter Einbezug des Systems von John Brown (1735-1788), cf. Janssen. l. c.

44 Karl August Feierabend (1812-1887?), gebürtig aus Stans, übte seinen ärztlichen Beruf in Hochdorf, Kappel (Toggenburg) und Luzern (1859-1882) aus. Er soll auch als Schriftsteller und Volkskundeforscher wissenschaftlich aktiv gewesen sein.

45 «[...] Vereinzelte Fälle jedoch kommen alljährlich in verschiedenen Gegenden vor.» ef. Feierabend. op. cit. p. 107.

46 v. Baas, Johann Hermann: Grundriss der Geschichte der Medicin und des heilenden Standes. Stuttgart: Enke, 1876. p. 585.

47 cf. exemplarisch zur entsprechenden, zeitgebundenen Konstitutionseinteilung für das 18. Jh.: Ilmoni, Immanuel: Bidrag till Nordens Sjukdoms-Historia. Tredje Delen. Helsingfors: Simeli, 1853. pp.404-407; v. Haeser, Heinrich: Lehrbuch der Geschichte der Medicin und der epidemischen Krankheiten. Nachdr. d. 3. Aufl. (Jena 1882). Hildesheim: Olms, 1971. Bd.3: Geschichte der epidemischen Krankheiten. pp.379-382, 418-419, $513-514$.

48 op. cit. p. 531.

49 v. Wagner. op. cit. p. 211.

50 v. Höfler, Max: Deutsches Krankheitsnamen-Buch. München: Piloty \& Lochle, 1899. pp. 678-680.

51 z. B. «Lenzen-Adl〉, 〈Stick-Adl〉, «Lausanner Fieber), (neue Krankheit〉, 〈bösartige StichKrankheit), (Pleuresch〉, (galliger/hitziger/pestilenzischer Seitenstich〉, cf. Höfler. op. cit. pp. 2, 142, 320, 323, 474, 678-680.

52 op. cit. p. 678.

53 cf. Andree, Richard: Trudensteine. in: Zeitschrift des Vereins für Volkskunde 13 (1903): 295-298. Er schreibt u. a.: «Ferner fand der Stein [= Trudenstein. Anm. d. Verf.] in der Volksmedizin Verwendung gegen den Stich, der im Volksmunde auch Alpstich heisst und eine Art Pneumonie ist, vom Alpdämon verursachter stechender Schmerz, der die Atmung so behindert, dass Erstickungstod eintritt.» (p.297); dasselbe berichtet (auf col.1175): Olbrich, Karl: Trudenstein. in: Handwörterbuch des deutschen Aberglaubens/hrsg. v. Eduard Hoffmann-Krayer. Berlin: de Gruyter, 1936/37. (Handwörterbuch zur deutschen Volkskunde; Abt. 1: Glaube). Bd.8. coll.1174-1176.

54 v. Sudhoff, Karl: Lungenentzündung. in: Reallexikon der Germanischen Altertumskunde/hrsg. v. Johannes Hoops. Straßburg: Trübner, 1915-1916. Bd.3. p. 170.

55 Zu wirkungsgeschichtlicher Analogie v. Püschel, Erich: Die «Varen» in der medizinischen Literatur seit Johann Weyer (1515-1588). in: Medicinae et artibus: Festschrift für [...] Wilhelm Katner zu seinem 65.Geburtstag. Düsseldorf: Triltsch, 1968. pp. 110-120.

56 Sticker, Georg: Beschreibung eines Schädels mit veralteter traumatischer einseitiger Unterkieferverrenkung. Ein Beitrag zur Lehre von den mechanischen Formveränderungen der Knochen. Bonn, Univ., Med. Fak., Diss., 1884.

57 Id.: Magensonde und Magenpumpe. in: Deutsche Medizinal-Zeitung 5 (1887): 141-[...]-227; Id.: Die Bedeutung des Mundspeichels in physiologischen und pathologischen Zuständen. in: Deutsche Medizinal-Zeitung 7 (1889): 1-[...]-212; u.v.a. 
58 Id.: Die semiotische Bedeutung des Fredericq-Thompson'schen Zahnfleischsaumes in der tuberculösen Phthise. in: Münchener Medicinische Wochenschrift 35 (1888): 618-620; Id.: Die Behandlung der Lungenschwindsüchtigen. Würzburg: Stahel, 1893; u.a.

59 Id.: Der Keuchhusten. Der Bostock'sche Sommerkatarrh (Das sogenannte Heufieber). Wien: Hölder, 1896. (Specielle Pathologie und Therapie; 4, 1, 3).

60 Id.: Mittheilungen über Lepra nach Erfahrungen in Indien und Ägypten. in: Münchener Medicinische Wochenschrift 44 (1897): 1063-1065; Id.: Ueber die Pest nach Erfahrungen in Bombay. in: Münchener Medicinische Wochenschrift 45 (1898): 11-16.

61 Id.: Die neue Kinderseuche in der Umgebung von Giessen (Erythema infectiosum.). in: Zeitschrift für praktische Aerzte 8 (1899): 353-358.

62 Id.: Die Entwicklung der ärztlichen Kunst in der Behandlung der hitzigen Lungenentzündungen. Wien: Hölder, 1902. p. 69.

63 v. Leyden. op. cit. p. 283.

64. Exemplare existieren meiner Kenntnis nach für die Schweiz nur in Lausanne (Bibliothèque Cantonale et Universitaire) und für Großbritannien in London (The Wellcome Institute for the History of Medicine), während in Deutschland kein einziges in offentlichen Bibliotheken nachzuweisen ist.

65 Das Manuskript befindet sich im Nachlaß Sticker (G, 4 Alpenstich). - Die in der Orthographie leicht abweichende Druckfassung erschien in: 17 [Seventeen]th International Congress of Medicine, London: 1913 [Proceedings]. London: Frowde [u. a.], 1914. Section 23: History of Medicine. pp. 183-185.

66 Die Einleitung basiert sinngemäß auf der Arbeit von Feierabend.

67 cf. Sticker, Georg: Abhandlungen aus der Seuchengeschichte und Seuchenlehre. I: Die Pest. Gießen: Töpelmann. 1: Die Geschichte der Pest. 1908. pp.12, 103; 2: Die Pest als Seuche und als Plage. 1910. pp. 242-247, 255-258.

68 Bei den Pleuritiden dürfte es sich analog in erster Linie um symptomatische, selten um protopathische gehandelt haben.

69 Zur Frage der Lungenpest in historischer Beziehung v. Becht, Hans-Peter: Medizinische Implikationen der historischen Pestforschung am Beispiel des «Schwarzen Todes» von 1347/51. in: Stadt und Gesundheitspflege [...]. Sigmaringen: Thorbecke, 1982. (Stadt in der Geschichte; 9). pp. 78-94. Zur Identifizierung des (Alpenstichs〉 als Lungenpest v. Waldis. l.c. - Zusammenfassend heißt es dazu bei Koelbing, Huldrych Martin: Zur Geschichte der Pest in der Schweiz. in: Jahrbuch für Solothurnische Geschichte 57 (1984): 5-12, auf S.7: "Im Gegensatz zu den Epidemien der Beulenpest, die von unseren Vorfahren eindeutig erkannt und beschrieben wurden, blieb den Zeitgenossen der wahre Charakter der Ausbrüche primärer Lungenpest verborgen; und man sprach von (Stich〉 (wegen des stechenden Brustfell-Schmerzes), 〈Lungensucht〉, 〈pestilenzischen Flüssen〉 oder dergleichen.» - In Stickers später 〈loimologischer Formels wird die Lungenpest nicht erwähnt, die «anthropozentrische» Seuchenformel im Gegenteil abgelehnt, cf. Sticker, Georg: Die Pestgefahr, Rückblick und Ausblick. in: Die medizinische Welt 11 (1937): 33-35, 66-70.

70 «Pneumonien mit besonderer Ätiologie», d.h. Pneumokokkenpneumonie, Pestpneumonie, Lungenmilzbrand, Pneumotyphus, Psittakose, Grippepneumonien u.a., behandelt überblicksmäßig: DeLaCamp, Oskar: Die Lungenentzündungen. in: Spezielle Pathologie und Therapie innerer Krankheiten/hrsg. v. Friedrich Kraus. Berlin: Urban \& Schwarzen- 
berg. Bd.3 (1924). pp.138-294. - Des weiteren ef. Jahn. 1.c.; Nicolaier, Arthur: Milzbrand (Anthrax, Pustula maligna, Carbunculus contagiosus, Mycosis intestinalis). in: Handbuch der Praktischen Medizin/hrsg. v. Wilhelm Ebstein. Stuttgart: Enke, ${ }^{2} 1906$. Bd.4. pp.309-323; Lenhartz, Hermann: Erysipelas (Rose, Rothlauf) und Erysipeloid. Wien: Hölder, 1899. (Specielle Pathologie und Therapie 3, 3); Sticker (1902). op. cit.; Aufrecht, Emanuel: Die Lungenentzündungen. Wien: Hölder, 1899 (Specielle Pathologie und Therapie; 14, 1.2). pp.206-220.

71 Zusammenfassend zur «katastasis loimike» und zum «contagium vivum) v. Liebermeister, Carl: Ueber die Ursachen der Volkskrankheiten: E. akad. Antrittsrede, geh. in d. Aula d. Museums zu Basel am 22.Sept. 1865. in: Id.: Gesammelte Abhandlungen. Leipzig: Vogel, 1889. pp. 1-25.

72 v. Sticker, Georg: [Rez. zu:] Mittwoch, Eugen. Die älteste Influenza-Epidemie in Persien und Mesopotamien (i. J. 855 n. Chr.). Berlin. klin. Wochenschr. 1913. in: Mitteilungen zur Geschichte der Medizin und der Naturwissenschaften 12 (1913): 501.

73 ef. Guttmanns Medizinische Terminologie. 29. Aufl., hrsg. v. Herbert Volkmann. Berlin: Urban \& Schwarzenberg, 1939. col.28: «Alpenstich: Epidemische Pneumonie in manchen Alpentälern.»

74 cf. Rodenwaldt, Ernst: Pest in Venedig 1575-1577. Ein Beitrag zur Frage der Infektkette bei den Pestepidemien West-Europas. in: Sitzungsberichte der Heidelberger Akademie der Wissenschaften: Mathematisch-naturwissenschaftliche Klasse: Jg. 1952, Abh.2. Heidelberg: Springer, 1953; Zaddach, Bernd Ingolf: Die Folgen des Schwarzen Todes (1347-51) für den Klerus Mitteleuropas. Stuttgart: Enke, 1971. (Forschungen zur Sozialund Wirtschaftsgeschichte; 17); Müller, Paul: Die Pestepidemien des 17. Jahrhunderts im Stande Solothurn. in: Jahrbuch für Solothurnische Geschichte 57 (1984): 13-194. [Zürich, Univ., Med. Fak., Diss., 1984]; Koelbing, Huldrych M.: Pestsperre am Gotthard: Der mailändisch-schweizerische Vertrag von 1585 im Lichte neuerer Forschungen. [...]. in: Praxis: Schweizerische Rundschau für Medizin 75 (1986): 228-231. - Sticker selbst hat allerdings weder die Bedeutung des Menschenflohs (Pulex irritans) für die Pestübertragung in Frage gestellt [cf. Sticker (1908). op. cit. II: pp. 160-161], noch es unterlassen, auf die Ansteckungskette von Mensch zu Mensch nachdrücklich hinzuweisen [cf. ibid. pp.186-188, 221-223]. Der Grund für Stickers starres Festhalten an der (Formel) Ratte-Rattenfloh-Mensch liegt sicher in seinen diesbezüglichen Erkenntnissen aus Bombay begründet und in dem Umstand, daß er die verdiente Anerkennung für seine wegweisenden Pestforschungen außer im kleinen Historikerkreis nicht angemessen erhalten hat!

75 Zitat aus einem Manuskriptentwurf Stickers, heute in der UB Würzburg.

\section{Summary}

The terminus 'Alpenstich' (alpine epidemic pneumonia) was created in 1838 by the Swiss physician Johann Jakob Guggenbühl (1816-1863) who first described it in his monograph about that enigmatic infectious disease which afflicted especially the pulmonary region. With the help of biased and inaccurate historical research Guggenbühl gained doubtful testimony for the long existing illness and its threatening development. In spite of important arguments 
against his findings, brought by Conrad Meyer-Ahrens ten years later, the theory of the 'Alpenstich' kept being acknowledged as is shown, for example, by the third important study of that plague by August Feierabend. It was not until 1902 that a fundamental attack was launched by the German epidemiologist and medical historian Georg Sticker (1860-1960), who dared to seriously criticize his predecessor. His final report, given in London 1913, provided evidence that the so-called 'Alpenstich' never existed as an autonomous plague, except in the phantasy of Guggenbühl. In fact, all those epidemics which had been regarded as one and the same disease, generated from different causes and showed different symptoms. Sticker stressed the nexus between bubonic and pulmonal plague that used to follow each other in the seasons' run. Additionally, he made human anthrax, erysipelas, typhus and influenza mainly responsible for those countless epidemics with their high mortality rates which had been regarded as the mystical 'Alpenstich'.

Michael Quick

Institut für Geschichte der Medizin

der Universität Würzburg

Koellikerstraße 6 R

D-8700 Würzburg 